\title{
Modelo piloto de estudio del tratamiento rehabilitador de autoestima y comportamiento en adolescentes con dislexia y disortografía mediante su mejora lectoescritora
}

\section{Pilot Model of the Rehabilitating Treatment for Self-Esteem and Behavior in Adolescents with Dyslexia and Dysorthography by Improving their Literacy}

\author{
Linda Zuppardo $^{1 \mathrm{a}}$, Antonio Rodríguez Fuentes ${ }^{1 b^{*}}$ \& Francisca Serrano ${ }^{1 \mathrm{c}}$ \\ ${ }^{1}$ Universidad de Granada, Granada, España.
}

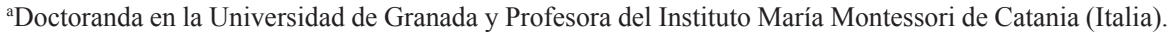
${ }^{b}$ Dr. en Ciencias de la Educación y Profesor Titular de la Facultad de Ciencias de la Educación de la

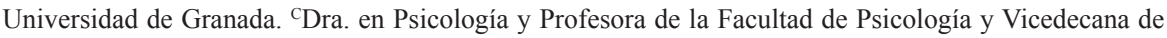
Investigación de la Universidad de Granada.

Recibido 05-05-17

Aprobado 26-07-17

En Línea 10-08-17

Correspondencia

Email: arfuente@ugr.es
Citar como:

Zuppardo L., Rodríguez Fuentes, A., \& Serrano F. (2017). Modelo piloto de estudio del tratamiento rehabilitador de autoestima y comportamiento en adolescentes con dislexia y disortografia mediante su mejora lectoescritora. Propósitos y Representaciones, 5(2), 359 - 400. doi: http://dx.doi. org/10.20511/pyr2017.v5n2.174

(C) Universidad San Ignacio de Loyola, Vicerrectorado de Investigación, 2017.

(cc) BY-NC-ND Este artículo se distribuye bajo licencia CC BY-NC-ND 4.0 Internacional (http://creativecommons.org/licenses/by-nc-nd/4.0/). 


\section{Resumen}

La investigación clínica y educativa ha puesto en evidencia los factores emocionales y motivacionales que caracterizan las dificultades de aprendizaje. El objetivo principal de este artículo es proponer un modo de valorar los efectos de un tratamiento rehabilitador en lectoescritura sobre la autoestima y sobre el comportamiento en un grupo de pacientes con diagnóstico de dislexia y disortografía. A un grupo de 60 estudiantes con diagnóstico de dislexia, en un primer momento, será suministrada una batería de test para valorar el perfil emotivo y conductual. Después, se dividirán a mitades un grupo control y otro grupo experimental que será sometido a un tratamiento rehabilitador para potenciar la lectura y la escritura. En síntesis, los resultados pretenden comprobar la hipótesis de investigación de que tras un adecuado tratamiento lectoescritor, con soporte de instrumentos y programas adecuados, los pacientes disléxicos pueden conseguir una mayor confianza en sí mismos y una consiguiente mayor autoestima, y no solo un desarrollo de la competencia comunicativa escrita.

Palabras clave: Autoestima, ansiedad, dislexia, disortografía, rehabilitación.

\section{Summary}

Clinical and educational research has shown the emotional and motivational factors involved in learning difficulties. The main objective of this research is to propose a way to assess the effects of a rehabilitating treatment for self-esteem and behavior through improving literacy in a group of patients diagnosed with dyslexia and dysorthography. Sixty students diagnosed with dyslexia will be given a test battery to assess the emotional and behavioral profile. Afterwards, they will be divided into a control group and experimental group, which will undergo a rehabilitating treatment to enhance reading and writing. In short, the results try to prove the hypothesis of research through an appropriate literacy treatment using appropriate instruments and 
programs. Patients with dyslexia can achieve greater self-esteem and not only a developed communication-writing competency.

Keywords: Self-esteem, anxiety, dyslexia, dysorthography, rehabilitation. 


\section{Introducción}

En los últimos años, se ha encontrado un aumento en la detección de personas con dificultades de aprendizaje. Las Dificultades Específicas de Aprendizaje (DEA) impiden el normal desarrollo de las habilidades específicas de la lectura, la escritura y el cálculo y pueden ser precedidos por un retraso en la adquisición del lenguaje oral. Se conocen como "específicos" porque se trata de una dificultad en el dominio específico de la habilidad, siendo el cociente intelectual igual o superior a la media. La Asociación de Dislexia Europea ("European Dyslexia Association": EDA) afirma que un promedio del 8\% de la población europea tiene dislexia (EDA, 2012). El porcentaje varía de país a país y depende de la trasparencia de la ortografía del idioma de referencia. La comunidad científica internacional considera la dislexia un trastorno congénito y genético, de origen neurobiológico, determinado por una alteración que obstaculizaría el desarrollo cognitivo de las funciones necesarias para la lectura, tales como la memoria, la percepción, la atención y la automatización de las funciones (Baddeley, 2000). Problemas característicos de la dislexia son la dificultad en la conciencia fonológica, memoria verbal y velocidad de acceso a material verbal. La dificultad de la lectura ocurre a menudo en comorbilidad con otros trastornos tales como disortografía (Ferraboschi \& Meini, 2005), disgrafía, discalculia, dificultades para almacenar definiciones de términos específicos, déficit de atención y problemas psicoafectivos (baja autoestima, ansiedad, trastornos de la conducta objeto de aprendizaje mutados, etc.) (Nicolson \& Fawcett, 2011).

\section{Variables emotivo-conductuales: autoestima y dislexia.}

La dislexia, que a menudo causa el fracaso en la escuela, con frecuencia se acompaña de problemas de conducta y emocionales: inestabilidad, indisciplina, resistencia, actitudes pasivas o agresivas, rechazo escolar, aislamiento y un sentimiento de inferioridad. Tales características, a menudo subestimadas, suponen un papel importante en la evolución del cuadro 
clínico. Los errores más comunes, como culpar al niño por el mal rendimiento escolar o atribuir la causa a problemas de carácter psicológico, a menudo repercuten en una mala imagen de sí mismo, en su confianza y en su baja autoestima (Chica, 2017). No sólo eso, las preocupaciones y las frustraciones de los padres ante las obvias dificultades de los niños, afectarían a la esfera emocional del niño con dislexia (Coltheart, 1998). Estos niños tienen a menudo historias de fracaso escolar, comprometiendo no sólo la escuela, sino también el desarrollo de la personalidad y su ajuste social (Keltikangas, 1992). Presentan ansiedad y frustración, que a veces conducen a depresión real y a dificultades de socialización. Estas cuestiones se convierten en aún más complejas en el periodo adolescente. De hecho, la adolescencia es una fase delicada de cambios e inseguridades, que determinan la personalidad del futuro niño (Rodríguez, Estévez \& Palomares, 2015), donde la autoestima es el primer elemento que se ve comprometido en los casos de fracaso escolar, y a su vez afecta al autoconcepto que incide en la motivación (Carranza \& Apaza, 2015). La autoestima, en efecto, se forma desde las primeras fases de escolarización, a partir de la comparación del propio rendimiento con lo esperado en distintos ámbitos de aprendizaje y la comparación con el rendimiento de los demás. Los jóvenes con baja autoestima presentan excesiva timidez e inseguridad en sus habilidades, miedo de cometer errores y a ser juzgados o no entendidos por otros. Esto conlleva a evitar la confrontación o el éxito potencial. Por lo tanto, es importante desarrollar una mayor confianza en sí mismos y en sus propias capacidades, sabiendo que cada uno tiene su propio valor. Así, si el niño puede lograr el éxito académico, "entonces su autoestima en la escuela será positiva" (Pope, McHale y Craighead, 1992, p. 16). Como resultado, la escuela contribuiría a la construcción de una autoestima y alta autoeficacia.

\section{Estudios previos.}

Con respecto a estos problemas psicoafectivos, estudios recientes han mostrado que los estudiantes con dislexia tienen niveles más bajos de 
autoestima, debido al mal rendimiento escolar y a la influencia que docentes, padres y compañeros de clase tienen en el desarrollo de la autoestima en las personas con dislexia, exacerbando el perfil de desempeño/logro (Humphrey \& Mullins, 2002a; Riddick, 1996). Algunos investigadores en este tema, como Riddick (1996) y Humphrey (2002), muestran que hay una escasez de investigación sobre el autoconcepto y la autoestima para alumnos con dislexia y es un tema que es necesario abordar.

La experiencia clínica y los datos de diversas investigaciones muestran, en efecto, que las DEA se presentan frecuentemente asociadas a problemas emocionales y conductuales, considerados un factor de riesgo para un futuro trastorno psicológico (Moè, De Beni \& Cornoldi, 2007; Mugnaini, Chelazzi \& Romagnoli, 2008).

Con respecto a sus compañeros sin DEA, las personas con dislexia tienen un concepto de sí mismos más negativo, se sienten menos respaldados emocionalmente, muestran más ansiedad (Riddick, Sterling, Farmer \& Morgan, 1999), muestran poca autoestima y tienden a abandonar la tarea a las primeras dificultades. Se presenta un círculo vicioso, elevados niveles de ansiedad y baja autoestima durante la lectura interfieren con el desarrollo de la tarea, con directas implicaciones a nivel de memoria de trabajo (Eysenck, Derakshan, Santos \& Calvo, 2007) y empeoramiento ulterior de la ejecución. La experiencia clínica y las investigaciones cualitativas indican que la presencia de dislexia puede determinar una mayor posibilidad de malestar psicológico, también, pero no sólo, porque las propuestas educativas pueden poner al niño en una posición de particular desventaja y estrés con respecto a sus compañeros (Ryan, 2006). Además, tal dificultad si persiste en el tiempo y no es abordada adecuadamente puede influenciar la futura adaptación psicosocial.

El DSM V (APA, 2013), entre los problemas asociados a la lectura, cuenta numerosas condiciones disfuncionales, muchas de tipo neuropsicológico (déficit de la percepción visual) del lenguaje, de la atención, de la memoria y del desarrollo motor y psicopatológico (Rodríguez, 2017), como ansiedad, a 
menudo asociada a la depresión, cuya presencia es documentada ampliamente entre personas con DEA (Riddick, 1996). Diversas investigaciones han evidenciado que los niños con dislexia presentan una menor autoestima escolar (Polychroni, Koukoura \& Anagnostou, 2006) y niveles de ansiedad clínicamente significativa (Nelson y Harwood, 2013).

En particular, la ansiedad es el más frecuente síntoma emocional en los casos de dislexia; algunos estudios (Nelson \& Harwood, 2011) evidencian la presencia de síntomas atribuibles a la ansiedad escolar en alrededor del $70 \%$ de los niños con dificultad de aprendizaje. Estudiantes con problemas específicos de aprendizaje pueden desarrollar formas extremas de malestar emotivo que se traducen en impotencia aprehendida (Seligman, 1975), responsable de la desmotivación que induce al individuo a inculparse a sí mismo de la situación en que se encuentra y a percibirse incapaz de aprender, emergiendo a menudo rasgos de depresión. En los últimos años, una conspicua literatura ha descrito el malestar socio-emotivo que acompaña a menudo las DEA en todas las edades, confirmando la utilidad de su identificación precoz y la aproximación multidimensional (Morris, Schraufnagel, Chudnow \& Weinberg, 2009).

La relación entre la percepción de sí mismo, la confianza en las propias capacidades y éxito escolar, en la mayor parte de los estudios, aparece vinculada con una correlación positiva (KeltiKangas-Jarvinen, 1992); en otras palabras, se cree que el aumento de la autoestima, aumenta el éxito escolar y viceversa. Burden (2008) localiza aspectos importantes relativos a la imagen de sí mismos en las personas con dislexia, mostrando que tendrán una más baja autoestima en el ámbito escolar con respecto de los normo lectores. Si los niños con dislexia mejoran las modalidades para afrontar y gradualmente superar sus dificultades, su concepto de sí mismos podría mejorar como consecuencia. 


\section{Hipótesis y objetivos.}

Sobre la base de las premisas teóricas descritas, el trabajo que se aconseja, basándose en la hipótesis de que niveles bajos de autoestima pueden ser una consecuencia de los DEA, es indagar la presencia de un perfil típico de personalidad de los chicos con diagnóstico de dislexia y averiguar si se puede llegar a un incremento de la autoestima con un adecuado tratamiento de las habilidades en la lectura y en la escritura, en tanto que competencias académicas básicas determinantes del desenvolvimiento escolar, es decir del éxito escolar y más aún del fracaso escolar. Más concretamente, se espera que con un tratamiento específico para mejorar las habilidades de lectura y de escritura y sus habilidades cognitivas relacionadas, como la memoria, se propicie el aumento de la autoestima. Es decir, que el contraste entre el grupo de control y el grupo experimental, después del tratamiento, evidencie una mejora de la autoestima en el alumnado del grupo segundo respecto del primero, que represente la optimización lectora y escritora como fomento y mejora de la autoestima.

Los objetivos de la intervención se encaminan a lograr que un tratamiento dirigido a las anteriores habilidades académicas básicas, con el apoyo de herramientas y programas adecuados, pueda:

- Aumentar la autoestima del alumnado con dislexia del grupo experimental;

- Reducir cualquier comportamiento de ansiedad, producido por la falta de competencia lectora y escritora o sus niveles bajos de desarrollo respecto del grupo-clase;

- Mejorar el rendimiento en lectura y comprensión, que previsiblemente se encuentren por debajo de la media;

- Desarrollar y recuperar las habilidades ortográficas en la escritura, bien que no hayan sido adquiridas, bien que hayan olvidado o confundido. 


\section{Método o planteamiento metodológico potencial del estudio}

Se explicita a continuación el recorrido posible para llevar a buen puerto esta experiencia de valoración y rehabilitación de la autoestima y la ansiedad a través de instrumentos determinados y su mejora a través de la rehabilitación indirecta de la competencia lectora y escritora en el aula escolar, a través de programas también señalados y descritos.

\section{Participantes.}

El grupo experimental propuesto debería estar compuesto por 30 adolescentes con diagnóstico de dislexia, que recibirán un trato rehabilitador. El grupo de control debería ser compuesto por 30 adolescentes con diagnóstico de dislexia, no sometidos a ningún tratamiento. Los alumnos son seleccionados de entre el registro que existe en la unidad de psiquiatría de un hospital que colabora con la investigación, con diagnóstico de varios años de dislexia y con otras características como las que se detallan a continuación para conseguir que los grupos sean, dentro de lo posible, homogéneos o mejor dicho equivalentes en cuanto a dichos criterios. La selección rigurosa de los participantes ha obrado según los siguientes criterios de inclusión: certificación del diagnóstico de una DEA, y ausencia de síndromes genéticos, enfermedades metabólicas, déficit neurosensorial, daños cerebrales, etc. La edad de los participantes con diagnóstico de dislexia, disortografía, entre 10 y 14 años, igualados por género, qué frecuentan la escolarización básica obligatoria. La elección de los mismos para conformar los dos grupos, grupo control y grupo experimental, se realizó de manera aleatoria de entre los alumnos con diagnóstico claro y definitivo de dislexia, y de entre ellos y de acuerdo con las evaluaciones previas (pretest) y los parámetros y que interesan para ser investigados se han diferenciado unos para el grupo control y otros para el experimental. De esta manera se ha garantizado la equivalencia de los grupos arriba demandada por la investigación de acuerdo con el tipo de diseño seleccionado. LA proporcionalidad de la muestra se 
resolvió contando con la misma cantidad de alumnos y con proporcionalidad en cuanto a sexos, edades y tiempos de diagnóstico.

\section{Diseño cuasi-experimental.}

Para llevar a cabo la investigación aconsejada se utilizaría un diseño de tipo cuantitativo, de tipo cuasiexperimental con grupo control y grupo experimental y medidas repetidas pretest-postest (Teddlie \& Tashakkori, 2009), que permita estudiar en profundidad los casos individuales y conocer en detalle los perfiles emocionales conductuales de los participantes con dislexia. Recogiendo la propuesta de McNulty (2003), el objetivo es definir una línea común de análisis entre la investigación cualitativa y aquella cuantitativa de los aspectos emotivos y afectivo-conductuales en los DEA, sugiriendo un análisis que pueda ser utilizado tato en el ámbito clínico como en el didáctico. La investigación permitirá observar en directo a los participantes, recogiendo los datos, mediante el empleo de formularios y hojas de registro observacional participante y, después, a través de un enfoque cuantitativo, proyectar en diagramas y gráficos los resultados recogidos de modo que sean fácilmente visibles. A tal objetivo, el formulario se configura como anillo de conjunción entre la recogida de datos y sus análisis posteriores, pudiendo contar con estadísticos específicos. Ello, en efecto, tiene la característica de presentar preguntas idénticas a todos los sujetos implicados, de modo tal que las informaciones recogidas sean comparables entre ellos y las únicas variables sean los experimentados y las experiencias personales, que proveen puntos de vista diferentes. Además, el formulario, no estando atado a ninguna teoría específica, permite valorar y analizar los resultados con base en teorías y objetivos diferentes y puede ser usado junto a otros instrumentos. Resulta, por lo tanto, un buen punto de salida por una primera valoración global. 


\section{Procedimiento}

El presente trabajo se articularía en tres fases, de la duración total de 12 meses. Inicialmente los participantes serán localizados a los dos grupos, experimental y de control, con diagnóstico de dislexia y disortografía.

- Fase1. La primera fase de la búsqueda es dirigida a ambos los grupos, experimental y de control, y prevé una valoración inicial de la autoestima y eventuales otras manifestaciones psicopatológicas, de la duración de 3 meses aproximadamente, mediante el empleo de los siguientes instrumentos:

- Test de Valoración Multidimensional de la autoestima ("Test di valutazione dell'autostima": TMA) de Bracken (1993), se basa en el presupuesto de que la autoestima es un esquema conductual y cognitivo que se desarrolla según los principios del aprendizaje. La autoestima es considerada como una valoración que el individuo tiene sobre él mismo, sobre la base de las continuas retroalimentaciones ambientales, de los éxitos y fracasos y de las relaciones establecidas con los otros. La autoestima, según el autor, puede ser considerada, por tanto, como un mecanismo de respuesta, aprendido en el tiempo, que refleja la autoeficacia del individuo con un valor predictivo con respecto a comportamientos futuros. El TMA está basado en un modelo jerárquico de la autoestima, dentro del cual se asume que las muchas dimensiones de que está compuesta, asociadas a los múltiples contextos en que el individuo actúa, resultan de igual importancia en su contribución a la construcción general de la autoestima. El instrumento, compuesto por 150 ítems evaluados sobre escala tipo Likert de 4 puntos1, está constituido por afirmaciones positivas y rechazos. Los ítems de valor positivo son valorados en orden decreciente2; los ítems de valor negativo son valorados en orden 
creciente3. La prueba está estructurada en seis escalas, que coinciden con las dimensiones consideradas constitutivas de la autoestima, más una escala total, que provee informaciones sobre el juicio general que los participantes con dislexia tienen de sí mismos, a saber:

- Relaciones interpersonales;

- Emotividad;

- Competencia/Control sobre el entorno;

- Éxito Escolar;

- Vida Familiar;

- Experiencia corporal;

- Escala Total.

- Test Multidimensional de escalas de ansiedad para niños ("Multidimensional Anxiety Scale for Children": MASC) de March, Parker, Sullivan, Stallings y Conner (1997), es un formulario útil para indagar los síntomas ansiosos en los participantes de 8 a 19 años. Está compuesto por 39 preguntas de respuesta múltiple y permite determinar si el niño padece de un cuadro patológico de ansiedad (de acuerdo con los criterios del DSM). El formulario induce al niño a dar informaciones acerca de sus pensamientos, sentimientos y comportamientos. El sujeto contesta a las preguntas, rodeando el número que exhibe cuánto de verdadera sea la afirmación para él4.

Los datos conseguidos permiten plasmar seis tipos de informaciones:

- Síntomas físicos;

- Evitación;

- Ansiedad social; 
- Ansiedad de separación;

- Puntuación Total;

- Índice de ansiedad ("Anxiety Disorder Index": ADI). Este ADI indica cuál será la tendencia del niño hacia la ansiedad. Una puntuación alta tiene valor predictivo con respecto de la posibilidad de desarrollar en el futuro síntomas de ansiedad.

- Formulario sobre el comportamiento del niño ("Child Behavior CheckList": CBCL) para edades de 6 a 18 años, de Achenbach (2001), es una de las escalas de valoración del comportamiento que se utiliza a nivel internacional en el ámbito médico y de investigación de búsqueda. La escala permite indagar las competencias sociales y los problemas emotivo-conductuales de niños y adolescentes. El CBCL es un formulario rellenado por los padres, estructurado en dos partes. La primera parte recoge informaciones sobre varias áreas del funcionamiento personal y social, mediante preguntas sobre la participación en deporte, grupos, asociaciones, sobre las amistades, sobre el desempeño en pequeñas faenas, sobre las relaciones con otras figuras familiares y sobre el rendimiento escolar. Cuenta, además, con preguntas abiertas sobre eventuales enfermedades, dificultades de la persona evaluada, preocupaciones por algún aspecto en el crecimiento del hijo y sobre los aspectos positivos del niño. La segunda parte contiene 113 ítems que se presentan bajo forma de afirmaciones relativas a los comportamientos en varios ámbitos y los problemas relacionales. Los padres contestan a cada ítem atribuyendo una puntuación de frecuencia relativa al comportamiento evidenciado5. Dadas las puntuaciones, confrontadas con los valores normativos, se sacan dos puntuaciones totales, una por competencias, actividad, sociabilidad, rendimiento escolar y 
otra por los problemas emotivo-conductuales. Así, pues, aporta dos perfiles diferentes: perfil de competencias y perfil psicológico y/o psicopatológico. Los índices de autenticidad de este formulario son excelentes (rango $=0.85$ - 0.88) (Dedrick, Greenbaum, Friedman, Wetherington \& Knoff, 1997). Es uno de los instrumentos más utilizado en el campo de la investigación, contando más de 4000 publicaciones, de comprobada validez y fiabilidad. Muchos estudios han demostrado como el CBCL representa un instrumento válido y confiable para la valoración de los problemas emotivo-conductuales de los niños y los adolescentes, también en el contexto italiano (Frigerio, Cozzi, Pastore, Molteni, Borgatti \& Montirosso, 2006).

- Fase 2. La segunda fase de la investigación está dirigida al grupo experimental (20 alumnos con diagnóstico clínico de dislexia y disortografia) y prevé un tratamiento rehabilitador, de una duración total de unos 6 meses, dividida en 3 pasos 2 meses cada uno, que aspira a mejorar las prestaciones en la lectura, en la escritura y en la comprensión del texto, mediante el seguimiento de los siguientes pasos:

- Paso 1. Tratamiento sublexical: Software "Dislexia y tratamiento sublexical" (Cazzaniga, Re, Cornoldi, Poli \& Tressoldi, 2005), para desarrollar y mejorar las habilidades de lectura, aplicando el método sublexical. Este tratamiento es realizado por la presentación en el ordenador de ítems para leer, con facilitaciones para evidenciar las sílabas. El software también prevé el programa Cloze, es decir, de una lectura rápida y temporizada de palabras, indicada para los participantes que presentan problemas específicos en la ruta visual de lectura.

- Paso 2. Recuperación fonológica, mediante el software "Actividad de recuperación sobre análisis fonológico, síntesis fonética y acceso léxico" de Savelli y Pulga (2006). Este programa facilita los procesos de comprensión del texto, supera el sistema clásico de la 
pregunta con respuesta de elección múltiple y se basa en la técnica innovadora de las lagunas.

- Paso 3. Recuperación ortográfica, mediante el software "Recuperación en ortografía, recorrido por el control consciente del error" (Ferraboshi \& Meini, 2005), que consiste en un programa de recuperación de las habilidades ortográficas, por actividades graduales que contemplan la adquisición de la conciencia del error y proveen estrategias para controlar los procesos de escritura.

- Fase 3. La valoración del perfil emotivo-conductual, por el TMA, el MASC y el CBCL, será efectuada, antes y después, a todos los participantes con diagnóstico de dislexia (en total 60), con la colaboración de los neuropsiquiatras infantiles y de psicopedagogos especializados en DEA. Por tanto, se culmina el proceso de investigación con la fase de reevaluación de las dimensiones que interesan al estudio, puestas de manifiesto más arriba, y con las pruebas también antes descritas.

\section{Análisis de los datos.}

Por cuanto concierne a los procedimientos analíticos, la primera operación estadística será de tipo descriptivo para analizar el curso de los datos; eso permite resumir los datos de los dos grupos, experimental y de control, ilustrándolos en distribución de frecuencia y calculando así la media y desviación estándar. En un siguiente momento, nos valdremos de la función de la estadística, denominada inferencial, que permite extraer deducciones con respecto de la probabilidad que los datos conseguidos, sean debidos al caso, en vez de a nuestra hipótesis o viceversa. Motivo por el que tendríamos nuestra hipótesis nula $\mathrm{H}_{0}$, que se refiere a la causalidad de los resultados conseguidos, en otras palabras: los datos observados no serán significativamente diferentes de los que se conseguirían si los grupos fueran pertenecientes a una única población; $\mathrm{y}$, al contrario, la hipótesis alternativa $\mathrm{H}_{1}$ que refiere la no causalidad de los resultados: los datos observados serían 
significativamente diferentes de los que se conseguirían por casualidad. Serán empleadas herramientas estadísticas ad hoc, como Systat, SPSS, PSPP, EZanalyze, R o similares, para conseguir datos cuanto más verosímiles a la realidad indagada. Será respaldado, en esta fase metodológica, de una esmerada búsqueda bibliográfica que nos servirá de soporte para la elección de las técnicas estadísticas que serán puestas en marcha y que mejor se conformarán con el objeto de investigación. Concretamente, sobre la base de las experiencias pasadas, el estadístico deseable para este tipo de datos es el "t de Student" para muestras independientes, los análisis de las correlaciones de Pearson y, como colofón, la regresión lineal mediante el potencial de determinación o predicción y, en caso de ser notable, su consecuente ecuación de regresión.

\section{Conclusión, a modo de DAFO o FODA o SWOT, y posible discusión}

Se realiza una valoración inicial del proyecto como tal, de acuerdo con la técnica DAFO (Debilidades, Amenazas, Fortalezas y Oportunidades), conocida en el ámbito iberoamericano como FODA, derivado del contexto norteamericano bajo las siglas SWOT (Strengths, Weaknesses, Opportunities and Threats). En esta línea, considerada la escuela es el entorno fundamental en que los chicos con dislexia manifiestan las mayores dificultades es necesario que los estudiantes puedan mejorar las mismas prestaciones y competencias, para poder experimentar el éxito y no sentirse diferentes de sus coetáneos. Constituye, pues, tal argumento una fortaleza evidente e incuestionable del proyecto.

En efecto, Scott (2004) sustenta que el modo mejor de combatir la baja autoestima se consigue mejorando la capacidad de aprendizaje de un estudiante. Paralelo a ello está la cuestión del aumento de la motivación (Carranza \& Apaza, 2015). Por consiguiente, el aumento de confianza y autoestima de un chico disléxico tendrá un efecto positivo sobre su rendimiento, sobre su comportamiento social y sobre su actitud con respecto a las pruebas de la vida, a los éxitos y a las quiebras. Este proyecto se 
propone el objetivo de entender si un tratamiento rehabilitador, sobre un grupo de pacientes con diagnóstico de dislexia y disortografía, puede tener efectos positivos sobre la autoestima y sobre el comportamiento de los adolescentes, no sólo en el ámbito escolar sino en las muchas áreas de vida de los participantes. Se quiere, pues, confrontar los valores de la autoestima y el perfil emotivo-conductual, realzado por el suministro de los test (TMA, CBCL y MASC, antes descritos), entre el grupo de control y el experimental, antes y después del tratamiento, para valorar la evolución de las mismas. Existen, no obstante, numerosas amenazas que acechan tanto la valoración objetiva del progreso como la aplicación del tratamiento, en tanto que no se trata de un proyecto experimental sino cuasiexpermiental, en el cual el control de variables extrañas se antoja más dificultoso.

El tratamiento prevé, también, la implicación de la familia, que debe ser informada constantemente e implicada, también en la compilación del formulario sobre el comportamiento del niño (CBCL), realizando una eficiente colaboración entre padres y figuras profesionales especializadas. Esta dimensión se concibe a la vez como una debilidad del proyecto, por cuanto, no contamos con las evidencias necesarias de que las familias estén dispuestas y preparadas para la intervención adecuada, como de una orientación necesaria para el logro de los objetivos de este trabajo y todos los que tengan vínculo con el aprendizaje y escolarización del alumnado, como premisa básica de desarrollo del alumnado con dislexia. 
En síntesis, se pretende sustentar que un tratamiento completo y adecuado aplicado a los sujetos disléxicos conduce no sólo a la optimización de las habilidades de lectura, escritura y comprensión del texto, sino también de la autoestima general de los chicos y eventuales comportamientos ansiosos e inadaptados en relación con la escuela y con los procesos de aprendizaje. La orientación sobre cómo se ha de desarrollar este trabajo y la fortaleza de sus logros merecen el esfuerzo que se ha de emprender, y la superación de las amenazas y control de debilidades. Aun no se disponen de resultados finales puesto que se estén en periodo aplicación del programa para mejorar la competencia lectora y escritora con propósito de descubrir si ello influye positivamente en la autoestima, respondiendo a la hipótesis de investigación alternativa o conveniente para esta investigación, tras rechazar la hipótesis nula.

No se han realizado estudios como el presente, según la revisión realizado en diversos motores de búsqueda internacionales, pero todo apunta a que la hipótesis de partida formulada en la medida de que aumentar la autoestima a partir de la mejora de la competencia lectora y escritora y su correspondiente optimización escolar sea viable, a juzgar por la conveniencia de estas competencias lectoescritoras en la población de alumnado sin dificultades específicas (Gallego \& Rodríguez, 2015; Rodríguez \& Gallego, 2017), como en el presente estudio: dislexia o disortografía, e incluso en alumnado con trastornos (Gallego \& Rodríguez, 2016) y discapacidades (Gallego \& Rodríguez, 2011) o con necesidades educativas especiales en general (García, Salvador \& Rodríguez, 2010). De ahí que la experiencia pueda ser empleada en otros contextos y con otros alumnos, empleando los instrumentos adaptados al entorno en que se desarrolle la investigación. Esta oportunidad es la justificación para la presentación del esqueleto del proceso evaluativo investigador que se presenta en esta ocasión. 


\section{Referencias}

Achenbach, T. M., \& Rescorla, L. A. (2001). Manual for ASEBA SchoolAge Forms \& Profiles. Burlington, VT: University of Vermont, Research Center for Children, Youth, \& Families.

American Psychiatric Association (2013). Diagnostic and Statistical Manual of Mental Disorders, 5th Edition. N.Y: American Psychiatric Association. https://doi.org/10.1176/appi.books.9780890425596

Baddeley, A. D. (2000). The episodic buffer: A new component in working memory? Trends in Cognitive Sciences, 4(11), 417-423. https://doi. org/10.1016/S1364-6613(00)01538-2

Bracken B. A. (1993). Test di valutazione dell'autostima. Trento: Edizioni Erikson.

Burden, R. (2008). Is dyslexia necessarily associated with negative feelings of self-worth? A review and implications for future research. Dyslexia, 14(3), 188-196.https://doi.org/10.1002/dys.371

Cazzaniga S., Re A. M., Cornoldi C., Poli S., \& Tressoldi P. (2005). Dislessia e trattamento sublessicale. Attività di recupero su analisi sillabica, gruppi consonantici e composizione di parole. Trento: Edizioni Erickson.

Carranza, R., \& Apaza, E. (2015). Autoconcepto académico y motivación académica en jóvenes talento de una universidad privada de Tarapoto. Propósitos y Representaciones, 3(1), 233-263. https://doi.org/10.20511/ pyr2015.v3n1.72

Chilca, M. (2017). Autoestima, hábitos de estudio y rendimiento académico en estudiantes universitarios. Propósitos y Representaciones, 5(1), 71127. doi: https://doi.org/10.20511/pyr2017.v5n1.145

Coltheart, M. (Ed.) (1998). Pure alexia (letter-by-letter reading). Hove, England: Psychology Press.

Dedrick, R. F., Greenbaum, P. E., Friedman, R. M., Wetherington, C. M., \& Knoff, H. M. (1997). Testing the structure of the Child Behavior Checklist/4-18 using confirmatory factor analysis. Educational and Psychological Measurement, 57(2), 306-313. https://doi. org/10.1177/0013164497057002009 
Eysenck, M. W., Derakshan, N., Santos, R., \& Calvo, M.G. (2007). Anxiety and cognitive performance: Attentional control theory. Emotion, 7, 336353. https://doi.org/10.1037/1528-3542.7.2.336

Ferraboschi, L., \& Meini N. (2005). Recupero in ortografia. Percorso per il controllo consapevole dell'errore. Kit con CD-ROM. Percorso per il controllo consapevole dell'errore. Kit. Con CD-ROM. Trenzo: Centro Studi Erickson.

Frigerio, A., Cozzi, P., Pastore, V., Molteni, M., Borgatti, R., \& Montirosso, R. (2006). La valutazione dei problemi emotivo comportamentali in un campione italiano di bambini in età prescolare attraverso la Child. Behavior Checklist e ill Caregiver - Teacher Report Form. Infanzia e Adolescenza, 5(1), 24-37, 2006.

Gallego, J. L., \& Rodríguez, A. (2015). Líneas de investigación sobre Educación Especial en España: un estudio bibliométrico (2006-2010). Revista de Ciencias Sociales, 21(2), 219-233. Recuperado de: http:// produccioncientificaluz.org/index.php/rcs/article/view/20003/19936

Gallego Ortega, J. L., \& Rodríguez Fuentes, A. (2015). Development of the Writing Skills of Students in Compulsory Education in Spain. The New Education Review, 42(4), 53-64. https://doi.org/10.15804/ tner.2015.42.4.04

Gallego Ortega, J. L., \& Rodríguez Fuentes, A. (2016). Características de la planificación de la escritura en estudiantes con Síndrome de Down. Revista Actualidades Investigativas, 16(1), 1-29. http://dx.doi. org/10.15517/aie.v16i1.22666

Gallego Ortega, J. L., \& Rodríguez Fuentes, A. (2011). La planificación de la composición escrita por alumnado con discapacidad visual: un estudio de casos. Revista de Educación, 356, 557-581. Recuperado de: http:// www.revistaeducacion.educacion.es/re356_23.html

García Guzmán, A., Salvador Mata, F., \& Rodríguez Fuentes, A. (2010). La investigación cualitativa en el desarrollo de la competencia escritora de escolares con necesidades educativas especiales, Revista Textos. Didáctica de la Lengua y la Literatura, 54, 71-78. Recuperado de: http:// textos.grao.com/revistas/textos/054-las-lenguas-del-mundo 
Humphrey, N. (2002). Self-concept and self-esteem in developmental dyslexia: implications for theory and practice [Online]. Recuperado de: http://self.uws.edu.au/Conferences/2002_CD_Humphrey.pdf.

Humphrey, N., \& Mullins, P. (2002). Personal constructs and attribution for academic success and failure in dyslexics. British Journal of Special Education, 29(4), 196-203. https://doi.org/10.1111/1467-8527.00269

Keltikangas-Järvinen, L. (1992). Self-esteem as a predictor of future school achievement. European Journal of Psychology of Education, 7(2), 123130. https://doi.org/10.1007/BF03172889

March, J. S., Parker, J. D. A., Sullivan, K., Stallings, P., \& Conners, C.K. (1997). The Multidimensional Anxiety Scale for Children (MASC): Factor structure, reliability, and validity. Journal of American Academy of Child and Adolescent Psychiatry, 36, 554-565. https://doi. org/10.1097/00004583-199704000-00019

McNulty, M. A. (2003). Dyslexia and the life course. Journal of Learning Disabilities, 36(4), 363-378. https://doi.org/10.1177/002221940303600 40701

Moè, A., De Beni, R., \& Cornoldi, C. (2007). Difficoltà d'apprendimento: Aspetti emotivo-motivazionali. In C. Cornoldi (a cura di), Difficoltà e disturbi dell'apprendimento (pp.253-271). Bologna: Il Mulino.

Morris, M. A., Schraufnagel, C. D., Chudnow, R. S. \& Weinberg, W. A. (2009). Learning disabilities do not go away: 20- to 25- year study of cognition, academic achievement, and affective illness. Journal of Child Neurology, 24, 323-332. https://doi.org/10.1177/0883073808324218

Mugnaini, D., Chelazzi, C., \& Romagnoli, C. (2008). Correlati psicosociali della dislessia: Una rassegna. Dislessia, 5(2), 195-210.

Nelson, J. M., \& Harwood, H. (2011). Learning disabilities and anxiety: A meta-analysis. Journal of Learning Disabilities, 44(1), 3-17. https://doi. org/10.1177/0022219409359939

Nicolson, R. I., \& Fawcett, A. J. (2011). Dyslexia, dysgraphia, procedural learning and the cerebellum. Cortex, 47(1), 117-127. https://doi. org/10.1016/j.cortex.2009.08.016 
Polychroni, F., Koukoura, K., \& Anagnostou, I. (2006). Academic self-concept, reading attitudes and approaches to learning of children with dyslexia: do they differ from their peers? European Journal of Special Needs Education, 21(4), 415-30. https://doi.org/10.1080/08856250600956311

Pope, A, Mc Hale, S., \& Craighead, E. (1993). Migliorare l'autostima. Un approccio psicopedagogico per bambini e adolescenti. Trento: Erickson.

Riddick B. (1996). Living with dyslexia. The social and emotional consequences of special learning difficulties. London: Routledge. https:// doi.org/10.4324/9780203432600

Riddick, B., Sterling, C., Farmer, M. \& Morgan, S. (1999) Self-esteem and anxiety in the educational histories of adult dyslexic students. Journal of Dyslexia, 5, 227-248. https://doi.org/10.1002/(SICI)10990909(199912)5:4<227::AID-DYS146>3.0.CO;2-6

Rodríguez, A., Estévez, M., \& Palomares, J. (2015). Tratamiento curricular de la imagen corporal, autoestima y autoconcepto en España. Propósitos $y$ Representaciones, 3(1), 9-70. https://doi.org/10.20511/pyr2015. v3n1.68

Rodríguez, A. (2017). Evaluación de dificultades lectoras escolares. En M. El Homrani, F. Pe-afiel y A. Hernández (cords.), Entornos y estrategias educativas para la inclusión social. Granada: Comares.

Rodríguez, A. y Gallego, J. L. (2017). Aprendiendo a escribir durante la ense-anza obligatoria, ¿deseo o realidad? Calidoscópio, 15(1), 106-125. https://doi.org/10.4013/cld.2017.151.09

Ryan, M. (2006). Problemi sociali ed emotivi legati alla dislessia. Dislessia, 3(1), 29-35.

Savelli, E., \& Pulga, S. (2006). Dislessia Evolutiva: attività di recupero su analisi fonologica, sintesi fonemica e accesso lessicale. Trento: Centro studi Erickson.

Scott, R. (2004). Dyslexia and Counselling. London: Whurr Publications.

Stella, G., (2000). Lo sviluppo cognitivo. Roma: Bruno Mondadori editore.

Teddlie, C. \& Tashakkori, A. (2009). Foundations of mixed methods research: Integrating quantitative and qualitative techniques in the social and behavioral sciences. Thousand Oaks, CA: Sage. 